\title{
IMPACTO DE CAPRINOS E OVINOS SOBRE A COMUNIDADE DE PLANTAS REGENERANTES NA CAATINGA
}

\author{
Dione J. Ventura ${ }^{1}\left[\right.$ [), Bráulio A. Santos ${ }^{4 *}$ (i) \\ 1 Universidade Federal de Pernambuco, Programa de Pós-Graduação em Biologia Vegetal, Av. Moraes Rego, s/n, Cidade Universitária, Recife-PE - Brasil. \\ 2 Universidade Federal da Paraíba, Centro de Ciências Exatas e da Natureza, Departamento de Sistemática e Ecologia. Cidade Universitária, Castelo Branco, \\ 58051900 - João Pessoa, PB - Brasil. \\ *Autorpara correspondência: brauliodse@ufpb.br
}

Recebido em 07 de fevereiro de 2020. Aceito em 29 de julho de 2020. Publicado em 31 de julho de 2020.

\begin{abstract}
Resumo - Caprinos e ovinos são herbívoros ruminantes de médio porte capazes de sobreviver em uma variedade de condições ambientais. Nosso objetivo foi quantificar os impactos de forrageio destes animais sobre a comunidade de plantas regenerantes no Parque Nacional do Catimbau (PARNA). Foram medidos os impactos dos animais em 20 parcelas permanentes de 50 x $20 \mathrm{~m}$. Em cada subparcela foi realizada a contagem do número de fezes e extensão de trilhas dos animais, densidade e o número de espécie de plantas lenhosas regenerantes. Houve uma correlação negativa entre a densidade de plantas e a extensão de trilhas e números de fezes. No entanto, estes indicadores de forrageio não se correlacionaram com a riqueza de espécies. A composição florística não variou ao longo do gradiente de forrageio, indicando que as parcelas pouco ou muito impactadas pelos caprinos e ovinos podem apresentar floras similares. Sugerimos que a ovinocaprinocultura extensiva no PARNA, possui um baixo custo ecológico para a flora lenhosa atual. É provável que o grande evento de degradação da vegetação lenhosa já tenha ocorrido com a introdução de grandes rebanhos bovinos, e que hoje, os caprinos e ovinos mantenham a Caatinga em estágios iniciais ou intermediários de degradação e regeneração.
\end{abstract}

Palavras-chave: Atividades Antrópicas; Plântulas; Herbívoros; Pecuária; Florestas Secas e Pastoreio.

\section{IMPACT OF GOATS AND SHEEP ON THE REGENERATING PLANT COMMUNITY IN CAATINGA}

Aвstract - Goats and sheep are medium-sized ruminant herbivores capable of surviving in a variety of environmental conditions. The objective of this work was to quantify the impacts of the foraging of these animals on the community of regenerating plants in the National Park of Catimbau (PARNA). The impacts of the animals were measured in 20 permanent plots of $50 \times 20 \mathrm{~m}$. In each subplot, the number of feces and the animal track length, density and the species number of regenerating woody plants were counted. There was a negative correlation between plant density and the length of trails and the number of feces. However, these foraging indicators did not correlate with species richness. The floristic composition did not vary along the forage gradient, this indicates the plots with less or more impacted by goats and sheep may show similar floras. We suggest that extensive sheep farming in the PARNA has a low ecological cost for the current woody flora. It is probable that the great event of degradation of woody vegetation has already occurred with the introduction of large cattle herds, and that today, goats and sheep maintain the Caatinga in early or intermediate stages of degradation and regeneration.

KEYwords: Human Activities; Seedlings; Herbivores; Livestock; Dry Forest and Grazing. 
IMPACTO DE LOS CAPRINOS Y OVINOS EN LA COMUNIDAD DE PLANTAS QUE SE REGENERAN EN LA CAATINGA

RESUMEN - Los caprinos y ovinos son herbívoros rumiantes de tamaño mediano capaces de sobrevivir a una variedad de condiciones ambientales. El objetivo de este trabajo fue cuantificar el impacto del forraje de estos animales en la comunidad de plantas que se regeneran en el Parque Nacional de Catimbau (PARNA). Los impactos fueron medidos en 20 parcelas permanentes de 50 x 20m. En cada subparcela fue realizado un conteo de número de heces y la distancia de los senderos de los animales, densidad y número de especies de plantas leñosas que se regeneran. Como resultado se obtuvo una correlación negativa entre densidad de plantas, distancia de los senderos y número de heces. No obstante, los indicadores de forraje no se correlacionaron con la riqueza de especies. La composición florística no varió a lo largo del gradiente del forraje, lo que indica que las parcelas poco o muy impactadas por los caprinos y ovinos pueden presentar floras similares. Por lo tanto, se estima que el impacto de la cría extensiva de caprinos y ovinos en el PARNA tiene un impacto ecológico poco significativo para la flora leñosa actual. Es probable que el principal acontecimiento de degradación de la vegetación leñosa ya haya ocurrido con la introducción de grandes rebaños bovinos, y que hoy, los caprinos y ovinos mantienen la Caatinga en etapas iniciales o intermedias de degradación y regeneración.

Palabras clave: Actividades Humanas; Plántulas; Herbívoros; Ganado; Bosques Secos y Pastoreo.

\section{INTRODUÇÃO}

Os problemas ambientais observados com o avanço da população humana sobre as florestas tropicais são evidentes em todo o mundo. Cerca de $80 \%$ da superfície terrestre já foi alterada por atividades antrópicas (Sanderson et al. 2002). Estas atividades se caracterizam pela conversão da floresta em diferentes tipos do uso do solo que modificam o ambiente e geralmente levam à perda da biodiversidade e serviços ecossistêmicos (Dregne 1986; World Resouces Institute 1992; Melo et al. 2010). As atividades agropecuárias são uma das principais fontes de degradação e fragmentação de muitos ecossistemas. A pecuária chega a ocupar hoje aproximadamente $45 \%$ da superfície da Terra; a produção de carne ovina representa, por exemplo, $72 \%$ da produção mundial em países em desenvolvimento (Herrero et al. 2009). A Europa seguida do continente americano e asiático são os maiores compradores de carne ovina, importando juntos 747,7 milhões de dólares em proteína animal ovina (Couto 2003). A ovinocaprinocultura extensiva é uma atividade considerada pela FAO (1993) como importante agente promotor de degradação ambiental, mas na verdade há uma grande escassez de dados empíricos locais que comprovem o alto custo ambiental da atividade, especialmente na Caatinga (Santo et al. 2012).

Os caprinos domésticos (Capra bircus) são herbívoros ruminantes considerados por alguns ambientalistas como "O herbívoro mais destrutivo" (Devendra e Burns 1983; King 1985). Sua criação é prática comum em regiões áridas e semiáridas (Chynoweth et al. 2013) e dependendo da intensidade de pastejo, podem ter impactos positivos ou negativos em processos biológicos-chave, em diferentes escalas espaciais e temporais (Rosenthal et al. 2012). De forma geral, os impactos diretos negativos de pastejo compreendem a remoção física do tecido vegetal através da alimentação seletiva, que envolve a perda de materiais fotossintéticos, nutrientes e carbono, tecidos meristemáticos, flores e sementes (Pickett \& White 1985; Crawley 1985). Além disso, se a planta for menor do que o herbívoro, o pisoteio dos animais não só causará danos ao tecido vegetal, devido à quebra dos ramos das plantas, como também poderá reduzir o seu crescimento, sobrevivência e reprodução (Crawley 1997).

Ao afetar negativamente o desempenho das plantas, os caprinos e ovinos podem alterar processos populacionais-chave como, natalidade, mortalidade, imigração e emigração, alterando potencialmente a 
abundância e distribuição das espécies de plantas consumidas. Em nível de comunidade vegetal, é provável que o pastejo destes herbívoros afete negativamente a riqueza de espécies, a biomassa e a composição taxonômica e funcional da comunidade de plantas (Arévalo et al. 2007, 2011, Fernández-Lugo et al. 2013). Consequentemente, processos ecossistêmicos como produtividade primária e ciclagem de nutrientes também devem ser afetados pelo pastejo desses ruminantes (Severson \& Debano 1991; Adler et al. 2001).

Em adição aos efeitos negativos dos caprinos e ovinos, outros fatores abióticos agem sinergicamente sobre as comunidades de plantas de ecossistemas áridos e semiáridos. Variações nas precipitações e nas propriedades físicas e químicas do solo, por exemplo, podem afetar as funções vitais das plantas devido às mudanças na disponibilidade de nutrientes e água (Haridasan 1992). Tais variações nas propriedades do solo ocorrem naturalmente devido a história geológica da área, mas em escalas espaciais menores também podem ser induzidas pelos herbívoros ruminantes (Al-Seekh et al. 2009). Numerosos estudos têm mostrado que o pastoreio destes animais causa a destruição da estrutura do solo e leva à compactação, ocasionando uma diminuição em carbono orgânico e nitrogênio (Shi et al. 2010). Em contrapartida, os excrementos de caprinos e ovinos podem tornar o solo mais fértil, já que as fezes desses animais possuem quantidades significativas de nutrientes (Haynes e Williams 1993).

A Caatinga já perdeu quase metade dos seus mais de $800.000 \mathrm{~km}^{2}$ de vegetação original. Atualmente esta região é considerada como uma das menos protegidas do país, tendo apenas 7,5\% das suas áreas protegidas por unidades de conservação (MMA 2014). Cerca de 27 milhões de pessoas habitam esta região, sendo considerado o ambiente semiárido mais populoso do mundo (IBGE 2010; INSA 2012). Dentre outras atividades, a ovinocaprinocultura é uma prática comum. De fato, o superpastejo por caprinos e ovinos, a agricultura itinerante e a retirada de lenha para subsistência são categorizados como os principais fatores de degradação deste ecossistema (Pereira Filho et al. 2013). Em 2011, o nordeste do Brasil apresentou 8,53 milhões de cabeças de caprinos, porém esse número caiu para 7,84 milhões em 2012 (IBGE 2012).

Este trabalho tem como objetivo quantificar os impactos da criação extensiva de caprinos e ovinos sobre a comunidade de plantas regenerantes no Parque Nacional do Catimbau, Pernambuco. Ao longo de um gradiente de forrageio, estimado como base na extensão de trilhas e no número de fezes deixadas pelos animais, esperase que áreas sob maior pressão pelos caprinos e ovinos apresentem menor densidade e riqueza de espécies de plantas lenhosas regenerantes. Espera-se também que áreas sujeitas a níveis similares de pressão pelos herbívoros apresentem composição taxonômica semelhante.

\section{Material e Métodos}

\section{Área de estudo}

O estudo foi conduzido no Parque Nacional (PARNA) do Catimbau, localizado em Buíque, Tupanatinga, Sertânia e Ibimirim, no agreste do Estado de Pernambuco. O PARNA possui uma área de $607 \mathrm{~km}^{2}$ e está situado

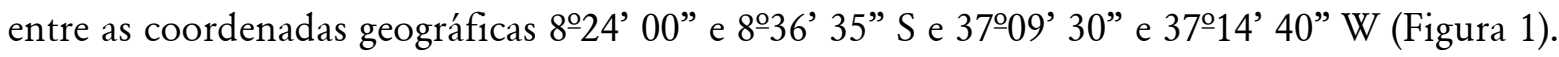


Figura 1 - Localização da área de estudo. Parque Nacional do Catimbau, município de Buíque, Pernambuco, Brasil.

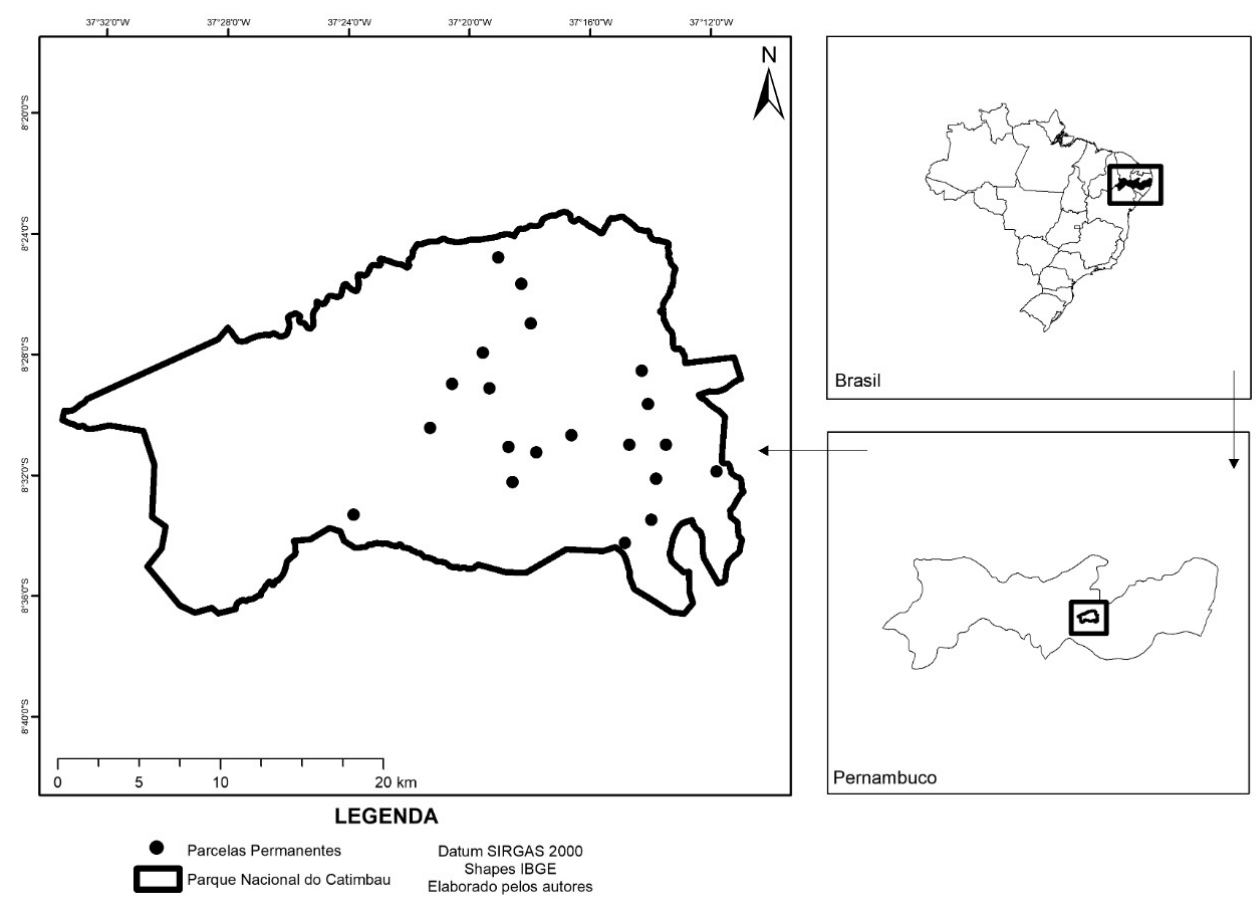

Tais municípios possuem criação extensiva de caprinos, principalmente Sertânia e Ibimirim, que estão incluídos entre os 20 municípios brasileiros com maiores rebanhos de caprinos do país (IBGE 2012). Embora o Parque tenha sido criado em 2002 pelo Decreto Federal 913/12, sua situação fundiária ainda é irregular. Não houve indenização dos antigos moradores, que continuam vivendo dentro dos limites da UC, havendo a presença de pequenos centros urbanos e pequenos criadores de caprinos. Estima-se que o parque possua uma densidade de $4 \mathrm{ind} / \mathrm{km}^{2}$ de caprinos (Jamelli 2015). Os diferentes usos do solo e pressão antrópica sobre a biota faz do Catimbau um local adequado para estudar como a ovinocaprinocultura extensiva afeta a biodiversidade da caatinga.

O clima predominante na região é o semiárido do tipo Bsh, com transição para o tropical chuvoso do tipo As', segundo escala de Köppen. Na região, as temperaturas médias anuais são de $23,7^{\circ} \mathrm{C}$ e precipitação média $706 \mathrm{~mm}$ ao ano, sendo o mês de maio o mais chuvoso e o de novembro o mais seco, com grande irregularidade no regime interanual (SADMET/INMET 2014). Geralmente, cerca de 60 a 75\% das chuvas ocorrem no período de março/abril até junho/julho.

Grande parte da área física do Parque compreende as movimentações do acidente orográfico conhecido como chapada de São José, em Buíque. Esta chapada foi formada pelo soerguimento de sedimentos cretáceos, depositados sobre depressões tectônicas do escudo cristalino, do Pré-Cambriano, dentro da bacia sedimentar da série Tucano-Jatobá, na área de drenagem do rio Moxotó (Jacomine et al. 1973). A geomorfologia do município onde está inserido o PARNA Catimbau é composta pela Bacia Sedimentar do Jatobá e sua área de entorno. É uma área de transição entre o ambiente cristalino e o sedimentar, ou seja, entre o planalto da Borborema e a bacia do Jatobá.

A área do PARNA Catimbau inclui diversas classes de solo. A predominância espacial corresponde às Areias Quartzosas (AQ), que ocupam 72,3\% do total da área. Os Planossolos (PS) estão situados na porção oeste, entre as cotas de 400 a 500 metros de altitude, correspondendo às vertentes suave onduladas da serra do Quiri d 'Alho, na área de drenagem dos afluentes do riacho dos Campos. Os solos Litólicos (R) compreendem os solos que acompanham as vertentes íngremes do paredão do cânion. Os Vertissolos (V) correspondem a solos argilosos das 
áreas de relevo plano e suave ondulado. E os Latossolos (LA) estão representados a oeste do Catimbau, em áreas de relevo suave ondulado e com altitudes de 800 a 1000 metros (Sociedade Nordestina de Ecologia et al. 2002).

A vegetação do Parque em determinadas áreas pode ser caracterizada por cinco ambientes com flora e vegetação distintas: caatinga arbustivo-arbórea; caatinga arbustiva com predominância de elementos de cerrado, caatinga arbustiva com elementos de campos rupestres, vegetação florestal perenifólia e caatinga arbustiva perenifólia (Rodal et al. 1998). Algumas localidades apresentam um tipo de solo arenoso e de cor esbranquiçada, que abriga várias espécies arbustivas típicas da caatinga arbustiva perenifólia: Croton micans, C. glandulosus, $C$. moritibensis, Jatropha ribifolia (Euphorbiaceae), Hyptis martiusii (Lamiaceae), Sida cordifolia, S. galheirensis, Herissantia crispa (Malvaceae). As espécies pertencentes às famílias das Fabaceae também estão presentes, tais como: Senna sp., Baubinia sp., Chamae crista sp., Crotalaria sp., Mimosa sp., Stylosanthes sp. e Poicinella microphylla. Algumas espécies arbóreas também são observadas, ressaltando-se as seguintes: Libidibia ferrea, Parapiptadenia zehntneri, Anadenanthera colubrina (Fabaceae), Schinopsis brasiliensis, Spondias tuberosa, (Anacardiaceae), ideroxylon obtusifolium (Sapotaceae), Cnidoscolus vitifolius e C. quercifolius (Euphorbiaceae).

\section{Desenho amostral}

Para mensurar o impacto dos ovinos e caprinos na regeneração das comunidades de plantas da caatinga, foram usadas 20 parcelas permanentes $(50 \times 20 \mathrm{~m})$, com distância de $2 \mathrm{~km}$ entre si, estabelecidas em solo predominantemente arenoso. Em cada parcela foram estabelecidas aleatoriamente (através de sorteio) três subparcelas de 5 x $5 \mathrm{~m}$. Durante janeiro e julho de 2014, todas as plantas lenhosas presentes nas subparcelas com diâmetro ao nível do solo (DNS) $\leq 2.9 \mathrm{~cm}$ e altura $\leq 1 \mathrm{~m}$ (Rodal, 1992) foram contadas e identificadas em nível de espécie. Em cada parcela também foi estimado o nível de forrageio pelos herbívoros ruminantes (caprinos e ovinos) a partir da extensão das trilhas e dos números de fezes deixadas pelos animais no interior de cada parcela. As contagens de fezes fornecem uma indicação potencialmente útil do número de animais numa determinada área (Landsberg et al. 1994). A extensão das trilhas (m) foi quantificada com uma trena de rodas, enquanto a quantidade de fezes foi contabilizada manualmente em 4 subparcelas, definidas aleatoriamente através de sorteio, de $5 \times 5 \mathrm{~m}$ distribuídas nas parcelas permanentes. Dado que a abundância e a distribuição das plantas podem ser influenciadas por outras variáveis ambientais, além dos caprinos e ovinos, em cada parcela também foram estimados os níveis de precipitação a partir de análise Geoestatística do ArcGIS, bem como a quantidade de matéria orgânica (M.O) e inorgânica do solo. No total, foram mensurados 10 parâmetros do solo $\left(\mathrm{pH}\left(\mathrm{H}_{2} \mathrm{O}\right), \mathrm{P}\right.$, $\mathrm{Ca}, \mathrm{Mg}, \mathrm{Na}, \mathrm{K}, \mathrm{Al}, \mathrm{H}$, água (\%) e M.O.

\section{Análise dos dados}

Os impactos dos caprinos e ovinos sobre a densidade e riqueza de plantas foram avaliados utilizando-se modelos lineares generalizados (GLM) (Crawley 2002). A densidade e riqueza de plantas regenerantes foram incluídas como variáveis dependentes e o número de fezes e extensão de trilhas dos herbívoros como variáveis independentes. Dado que as variáveis dependentes são discretas, a distribuição do erro foi ajustada para Poisson e a função de ligação para log, como recomendado por (Crawley 2002). Os modelos foram desenvolvidos no programa estatístico JMP8.

Para avaliar como as variáveis ambientais (matéria orgânica e inorgânica do solo, trilha, fezes e precipitação) influenciam a composição das espécies, inicialmente foi realizada uma Análise de Componente Principal (PCA) para reduzir o número de variáveis ambientais e julgar as variáveis mais importantes através da análise dos eixos. Para decidir quais eixos são mais representativos utilizamos o modelo nulo de vara quebrada; valores maiores do 
que estes modelos são mais representativos (Borcard et al. 2011). As variáveis que apresentaram correlações baixas nos eixos da PCA e as que apresentaram colinearidades foram retiradas para a análise subsequente. Em seguida, para avaliar quais variáveis ambientais influenciam a distribuição das espécies nas áreas estudadas, foi realizada uma Análise de Correspondência Canônica (CCA). Para tanto, os dados foram organizados em duas matrizes: uma contendo os dados de abundância das espécies nas parcelas e outra com os dados das variáveis ambientais transformados (ranging). Para avaliar a significância dos primeiros eixos da ordenação, empregou-se o teste de Monte Carlo (Ter Braak \& Prentice 1988), com 999 permutações. A riqueza e a abundância de espécies não fizeram parte desta análise, sendo utilizadas apenas como variáveis marcadoras. Todas as análises multivariadas foram realizadas no programa Fitopac 2.0 (Shepherd 2009).

Para avaliar o impacto dos caprinos e ovinos sobre a composição florística das comunidades estudadas foi utilizado um teste de Mantel Simples - rM (Legendre \& Legendre 1998), com 1000 permutações. O teste de Mantel é um procedimento estatístico de correlação entre duas matrizes (Mantel 1967) e leva em consideração a hipótese nula, ou seja, que há ausência de correlação linear entre as duas matrizes de distância (Legendre \& Legendre 1998). Para tanto, foram calculadas as distâncias euclidianas entre os pares de amostragem das matrizes florísticas e ambientais (fezes e trilhas).

\section{Resultados}

Foram registrados 1.783 indivíduos de plantas regenerantes de 27 famílias e 109 espécies (incluindo 9 morfoespécies), em 20 parcelas. O número total de excrementos e trilha foi de 10.170 fezes e $3.892 \mathrm{~m}$, respectivamente. Em média, foram registrados 89,15 indivíduos e 10,1 espécies de planta, 508,5 de excrementos e 194,63 metros de trilhas (Tabela 1). Também foi registrada uma precipitação de $681,4 \mathrm{~mm}$ e $15,21(\mathrm{~g} / \mathrm{kg}) \mathrm{de}$ M.O, em todas as áreas (Tabela 1). As famílias mais representativas foram Fabaceae (688 indivíduos e 33 espécies), Euphobiaceae (645 e 15), Cactaceae (155 e 2) e Boraginaceae (43 e 3). As espécies mais abundantes foram: Cnidosculos urens (175 indivíduos), Jatropha sp (162), Tacinga palmadora (153), Aeschynomene viscidula (113), Jatropha mutabilis (84), Croton biliotropiifolius (69) e Senna rizzini (57).

Tabela 1- Média dos Indicadores de perturbação dos caprinos e ovinos, indicadores ambientais e biológicos encontrados nas 20 parcelas permanentes localizadas no Parque Nacional do Catimbau, município de Buíque, Pernambuco, Brasil.

\begin{tabular}{lccc}
\hline & Média & DP & Min-Max \\
\hline Indicadores de perturbação & & & \\
Fezes & 508,5 & $\pm 537,3$ & $0-2201$ \\
Trilha (m) & 164,93 & $\pm 118,5$ & $0-426,6$ \\
Indicadores Ambientais & & & \\
Matéria Orgânica- M.O (g/kg) & 15,21 & $\pm 12,1$ & $4,48-47,7$ \\
Precipitação (mm) & 681,4 & $\pm 138,7$ & $510-940$ \\
Indicadores Biológicos & & & \\
№ de indivíduos & 89,15 & $\pm 128,2$ & $24-513$ \\
№ de espécies & 10,6 & $\pm 4,1$ & $5,0-21$ \\
\hline
\end{tabular}

A relação entre as medidas de impacto dos caprinos e ovinos (extensão de trilha e número de excrementos) não foi significativa, indicando que elas não são colineares $(r=0,28 ; n=20 ; p=0,83)$. Não houve efeito 
significativo do número de excrementos e extensão de trilhas sobre a riqueza de espécies regenerantes (Figura 2). No entanto, como esperado, o número de excrementos e a extensão das trilhas se relacionaram negativamente com a densidade de indivíduos, mas esta relação foi fraca (Figura3a, b; Tabela 2). A PCA da matriz ambiental mostrou um diagrama homogêneo (Figura 3). O percentual de variação acumulada nos dois primeiros eixos foi de 44,62\%, sendo maior do que o esperado pelo modelo nulo da vara quebrada (41\%). O primeiro eixo foi mais fortemente relacionado com Trilha $(0,56)$, precipitação $(-0,60)$ e $\mathrm{Al}(0,91)$; o segundo eixo foi mais relacionado com excrementos $(0,81)$ e K $(-0,51)$; e o terceiro com matéria orgânica $(0,65)$. Isso significa que essas variáveis foram utilizadas para a análise da CCA.

Figura 2 - Relação entre a densidade de indivíduos de plantas regenerantes e o log dos excrementos em (a) e o log das trilhas dos caprinos e ovinos em (b); relação entre a riqueza de espécies de plantas e o log dos excrementos em (c) e o log das trilhas dos caprinos e ovinos em (d), em 20 parcelas permanentes no Parque Nacional do Catimbau, município de Buíque, Pernambuco, Brasil.
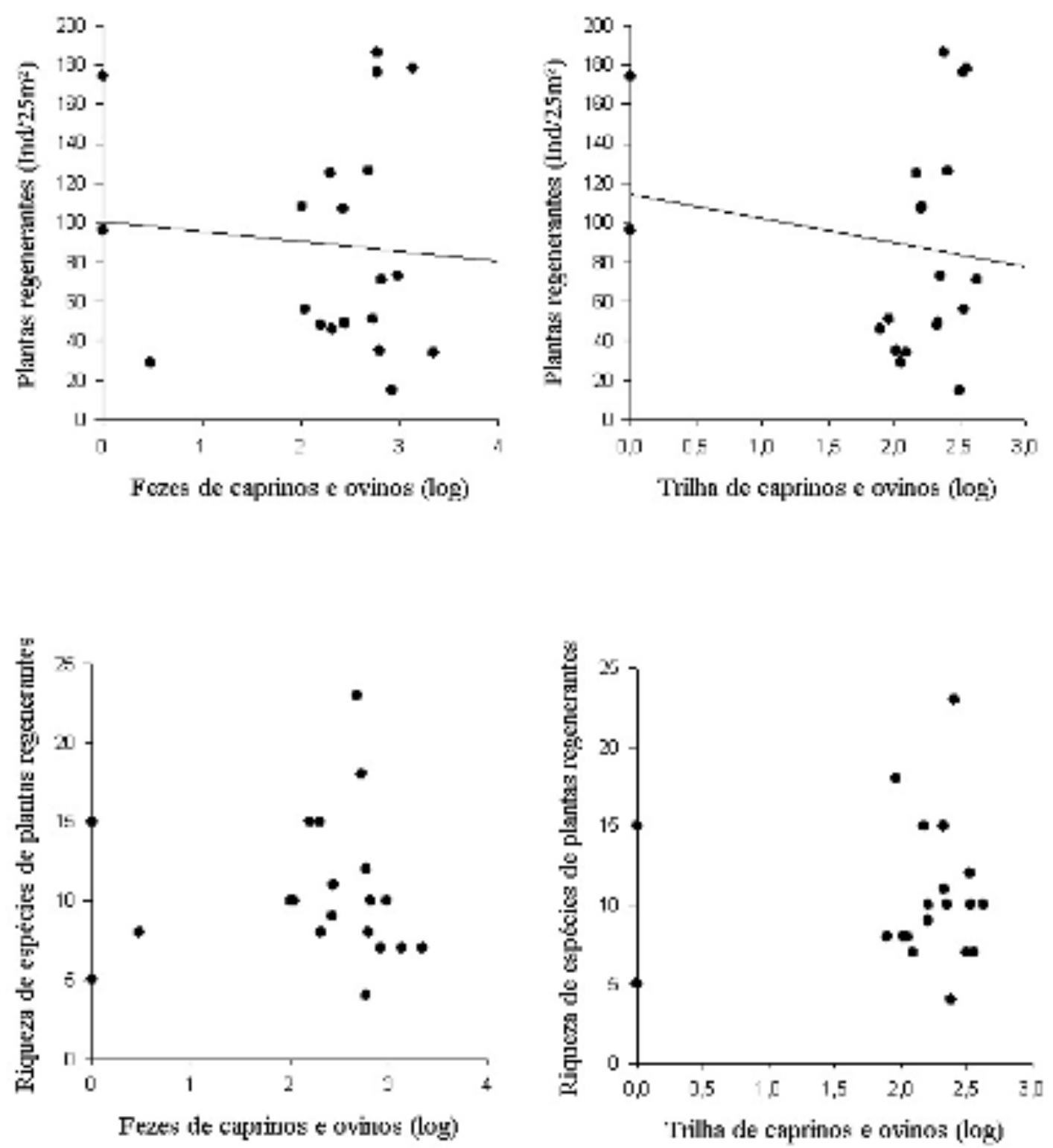
Figura 3 - Diagrama dos dois primeiros eixos da Análise de Componentes Principais (PCA) da matriz ambiental para as 20 parcelas permanentes no Parque Nacional do Catimbau, município de Buíque, Pernambuco, Brasil.

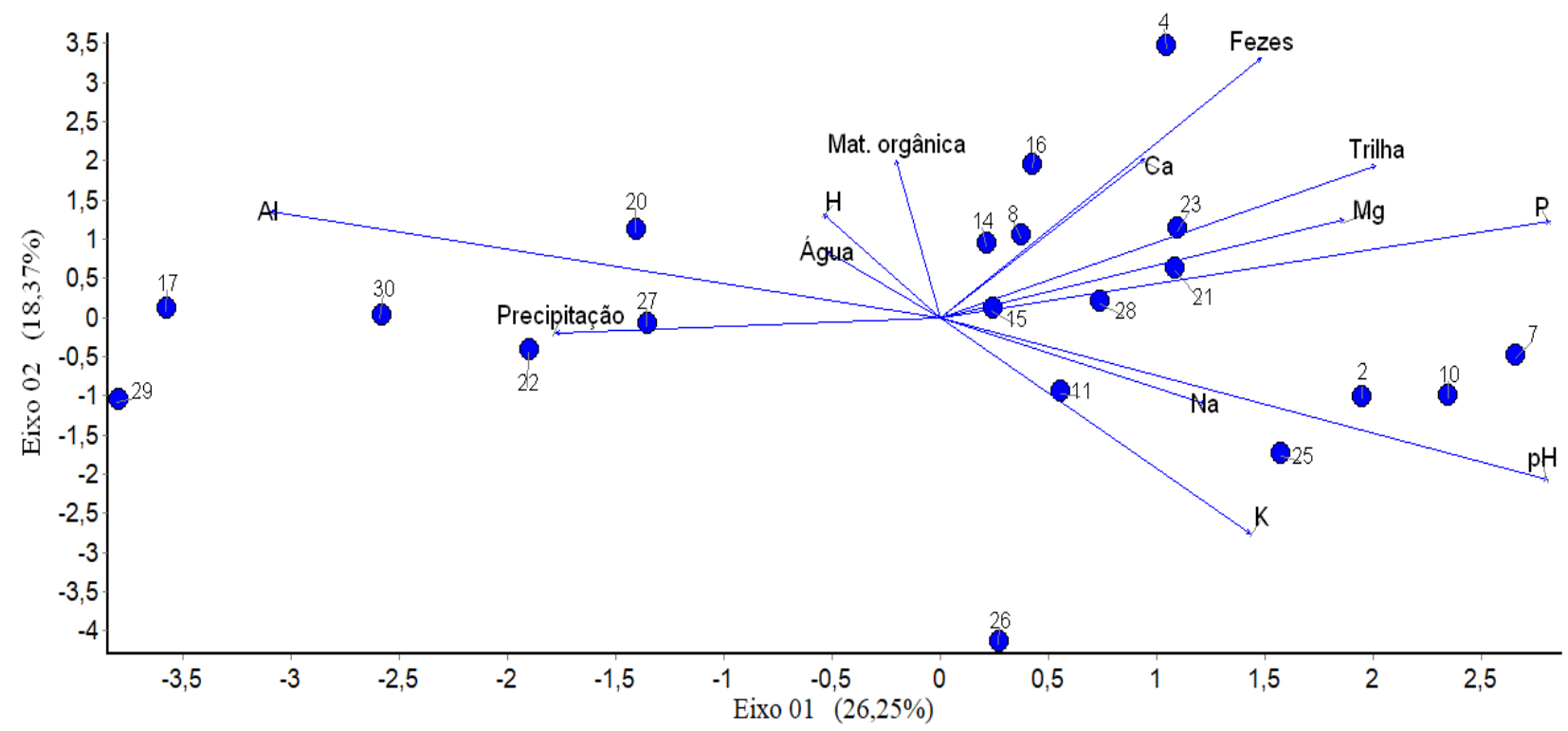

Tabela 2- Resultados dos Modelos Lineares Gerais (GLM) demonstrando os efeitos de perturbação dos caprinos e ovinos, sobre a riqueza e a densidade de indivíduos de espécies de plantas regenerantes em áreas de Caatinga, localizadas no Parque Nacional do Catimbau, município de Buíque, Pernambuco, Brasil. Valores em negrito destacam o efeito significativo $(\mathrm{p} \leq 0,05)$.

\begin{tabular}{lcccc}
\hline \multicolumn{1}{c}{ Atributos } & Variáveis explanatórias & Estimador & Valor $\mathbf{F}$ & Valores- $\boldsymbol{P}$ \\
\hline Riqueza de espécies & $\mathrm{N}^{\circ}$ fezes & 0,00 & 0,01 & 0,90 \\
& Trilha $(\mathrm{m})$ & 0,02 & 0,05 & 0,82 \\
Densidade de indivíduos & $\mathrm{N}^{\circ}$ fezes & $-0,05$ & 4,93 & $\mathbf{0 , 0 2}$ \\
& Trilha $(\mathrm{m})$ & $-0,12$ & 15,96 & $<\mathbf{0 , 0 0 1}$ \\
\hline
\end{tabular}

O diagrama de ordenação da CCA indicou que a riqueza e a abundância das espécies não seguem o padrão das variáveis ambientais em questão (Figura 4). Porém, as variáveis ambientais trilha, excrementos e M.O afetam igualmente a composição de espécies de algumas áreas; outras são mais influenciadas pela precipitação, alumínio e potássio. As variáveis ambientais que tiveram uma correlação forte com o primeiro e terceiro eixo (escores LC) foram, respectivamente, trilha $(0,62)$, M.O $(-0,81)$, e K $(0,71)$ e $\mathrm{Al}(-0,83)$. Os autovalores (AV) obtidos na análise para os três primeiros eixos da ordenação foram $0,66,0,59$ e 0,55 , sendo responsáveis respectivamente, por 7,2\%, 13,7\% e 19,8\% da variância total acumulada dos dados. As porcentagens de variância acumulada nos três primeiros eixos foram menores do que aquelas esperadas pelo modelo nulo da vara quebrada (AV1 esperado 21,4; AV2 40, 7 e AV3 58,8\%). Apesar dos eixos explicarem pouco sobre a distribuição da composição e as variáveis ambientais, estes são eixos interpretativos, pois o resultado dos testes de Monte Carlo foi significativo para o primeiro e terceiro eixo da ordenação (AV1 $p=0,03$ e AV3 $p=0,02)$. 
Figura 4 - Diagrama de ordenação do primeiro eixo com o terceiro eixo da Análise de Correspondência Canônica (CCA) para as 20 parcelas permanentes no Parque Nacional do Catimbau, município de Buíque, Pernambuco, Brasil. Cores denotam abundância e tamanho riqueza de espécies.

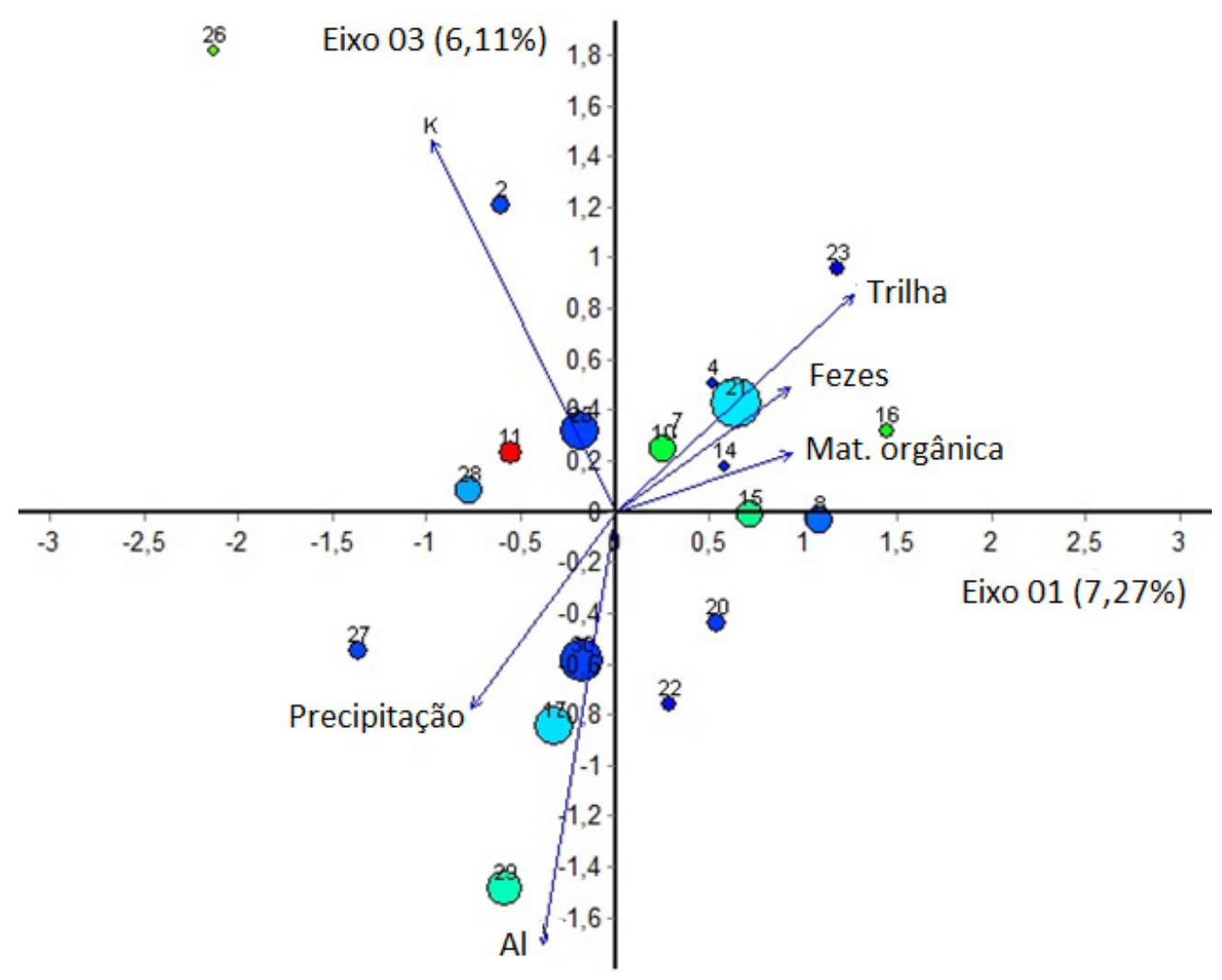

A previsão de que a composição de espécies de plantas seria influenciada pelo gradiente dos impactos ambientais dos herbívoros (trilha e excrementos) não foi confirmada, pois não foi observada uma correlação entre as matrizes de distância florística e a matriz ambiental de excremento $(\mathrm{rM}=-0,008 ; \mathrm{p}=0,55)$; nem entre a matriz de distância florística e a matriz ambiental trilha $(\mathrm{rM}=-0,07 ; \mathrm{p}=0,29)$.

\section{Discussão}

Embora os ovinos e caprinos tenham sido introduzidos na Caatinga há pelo menos quatro séculos, nenhum estudo havia estimado quantitativamente seu impacto sobre a estrutura e a composição taxonômica das comunidades de plantas regenerantes. Contrariando as expectativas, o presente estudo evidenciou um efeito neutro dos caprinos e ovinos sobre a riqueza de espécies e a composição florística da comunidade de plantas regenerantes no Parque Nacional do Catimbau. Embora a abundância de plantas tenha sido menor onde havia mais fezes e trilhas dos herbívoros, o conjunto geral dos resultados indica que a ovinocaprinocultura extensiva na área de estudo tem um baixo custo ecológico para a flora lenhosa atual. Esta afirmação, no entanto, precisa ser discutida em um contexto de tempo e espaço condizente com a realidade da atividade na região.

Trabalhos realizados em diferentes partes do mundo indicam que os caprinos e ovinos possuem um alto custo ecológico para a comunidade de plantas nativas. Recentemente, um estudo realizado no Parque Nacional do Catimbau observou que a exclusão pelos caprinos influenciou na diferenciação taxonômica de algumas espécies das comunidades herbáceas da área com efeito cumulativo ao longo do tempo (Menezes et al. 2020). Segundo os trabalhos de Tsuiki et al. (2005), realizado numa floresta de Pradarias na China, áreas que apresentavam pastejo leve e moderado exibiam riqueza de espécies de planta semelhantes, diferente do encontrado em áreas com pastejo 
pesado que mostraram menor riqueza e diversidade de espécies. Na África do Sul, um experimento de exclusão realizado numa floresta seca, revelou uma mudança na composição de espécies em áreas que possuíam caprinos (Cowling 1994). Já o estudo de Friedel (1986) verificou influência de ovelhas e coelhos na densidade de mudas de espécies arbóreas; na Austrália, vários estudos corroboram isto, nos quais mostram que a atividade de pastejo altera negativamente a densidade e o recrutamento de plantas lenhosas e transforma florestas em pastos (Gibson \& Kirkpatrick 1989, Williams 1990). Estudos simulando a herbivoria, em regiões mediterrâneas, indicam que o crescimento de mudas da espécie Quercus pyrenaica é limitado pela 'navegação' dos herbívoros (Baraza et al. 2004). De fato, muitos herbívoros ruminantes, tais como os caprinos, são generalistas e chegam a consumir cerca de 70\% das partes das plantas jovens, limitando a densidade de indivíduos, o crescimento e atrasando a maturidade reprodutiva das plantas (Leal et al. 2003, Barnes 2001).

Por outro lado, existem estudos que apresentam impacto neutro dos herbívoros sobre a comunidade de plantas e alguns grupos de vegetais conseguem sobreviver sobre pressão de pastejo. Ungulados em geral, incluindo caprinos e ovinos, evitam plantas que apresentam substância química, defesa física e certo grau de toxicidade, incapazes de serem degradados no trato digestivo (Forsyth et al. 2002). Várias plantas da caatinga, tais como Cnidosculus urens e Tacinga palmadora, são pouco palatáveis e possuem estratégias de defesa contra os animais herbívoros. O Cnidosculus, por exemplo, é uma planta cianogênica conhecida por causar intoxicação em muitos caprinos e bovinos (Riet-Correa et al. 2011). Devido a essas estratégias de defesas muitos animais evitam o contato com essas plantas, tornando-as mais abundantes nas áreas e preferindo forragear por áreas abertas. Apesar disto, registros de caprinocultores indicam que as cabras possuem habilidades digestivas para se alimentar de diferentes partes das plantas. Por exemplo, foi identificado que estes animais consomem plântulas, folhas novas e maduras, flor e fruto das espécies de Cnidosculus quercifolius (Leal et al. 2003). Pokorná et al. (2013) evidenciaram que não houve efeito da pressão dos caprinos sobre um grupo de plantas, onde o maior tempo de pastejo não influenciou a cobertura vegetal dos arbustos; o mesmo efeito neutro foi observado por Arévalo et al. (2011), sobre a riqueza de uma comunidade de plantas numa floresta Mediterrânea, na Espanha, corroborando os resultados encontrados em nossos estudos. Tais resultados evidenciam a necessidade de novos trabalhos para um melhor entendimento dos impactos dos caprinos sobre as comunidades de plantas.

Um estudo realizado na mesma área e no mesmo período desta pesquisa mostra que caprinos forrageiam preferencialmente em áreas de caatinga aberta (Jamelli 2015), provavelmente devido ao alto nível de degradação ambiental já existente na área, facilitando o forrageio destes animais sobre as plantas regenerantes como observado nas baixas densidades de plantas jovens encontradas para nosso estudo. Isso provavelmente ocorre porque as plântulas ficam mais expostas ao consumo, sendo o impacto direto desses animais mais fortes do que os indiretos, como: competição com gramíneas que são resistentes ao pastejo e o pisoteio que leva a compactação do solo, impedindo a infiltração de água e o estabelecimento de plântulas. Além disso, a maioria das plantas jovens possui folhas pouco rígidas e fibrosas quando comparadas as plantas mais velhas, o que aumenta a palatabilidade delas por esses animais, aumentando a mortalidade e levando ao baixo estabelecimento de plântulas (Augustine; Mcnaughton, 1998).

É possível ainda que estes animais pastoreiem sobre uma vegetação historicamente degradada pelos bovinos e pela exploração de lenha para atividades de subsistência da população (Melo 2017, Specht et al. 2019), no qual podem explicar os impactos neutros sobre a riqueza de espécies de plantas e a composição taxonômica da vegetação encontrada em nosso estudo. Apesar do diagrama da CCA demonstrar que algumas áreas apresentam um leve agrupamento da composição florística quando relacionados a variáveis ambientais de impacto dos herbívoros e a matéria orgânica do solo. Isso porque em áreas de pastejo, as excreções destes animais afetam as condições e a fertilidade do solo já que seus excrementos contêm quantidade significativa de nutrientes (Hakamata \& Hirashima 1978, Haynes \& Williams, 1993).

Assim, esses resultados que estão ocorrendo no Parque Nacional do Catimbau, pode ser um panorama representativo para a caatinga, visto que todo o bioma apresenta grande quantidade de herbívoros, principalmente 
caprinos. A caatinga há muito vem sofrendo com a pressão antrópica sobre sua biodiversidade. A princípio, no século XVII, foi à pecuária que teve papel fundamental no processo de interiorização, segundo relata Prado Junior (1981). Desde a chegada dos colonos que suas terras são utilizadas para a pecuária extensiva; à medida que os canaviais se expandiam no litoral, o gado era levado para o interior, e, dessa forma, talvez a grande perda de biodiversidade da caatinga tenha sido causada antes pelos bovinos, que devido ao seu maior tamanho corporal causam maiores danos no ambiente. Estes pisoteiam, quebram galhos e consumem mais vegetação que um animal ruminante de médio porte, como os caprinos. Hoje as cabras e ovelhas provavelmente mantêm a caatinga num estágio inicial de sucessão. Porém, esses animais fazem parte de um importante ativo econômico para a região, além de serem os ruminantes de médio porte que mais tem impactado a economia mundial dos últimos anos (Tedeschi et al. 2008). Atualmente, os produtores e os principais setores pecuaristas estão levantando mais esforços para aumentar a produção de leite e carne de caprinos em áreas nas quais esses animais possuem alta adaptação, como na caatinga (Santos et al. 2005). Isso vai além da economia, pois é de conhecimento que a carne caprina e ovina é de grande importância nutricional para a população, além de gerar emprego e renda para o homem sertanejo. Assim, a criação de cabras e ovelhas possui um alto benefício econômico e social para a caatinga. O grande desafio está em maximizar esses benefícios e reduzir os possíveis danos ecológicos que estes animais causam à biota nativa.

\section{Agradecimentos}

Este estudo foi apoiado pelo Conselho Nacional de Desenvolvimento Científico e Tecnológico (CNPq - Processo PELD 403770 / 2012-2). Agradecemos aos membros dos laboratórios LIPA e LEVA pela ajuda durante o trabalho de campo, principalmente, Davi Jamelli e aos, ainda, moradores do parque por permitir trabalhar na área. Agradecemos a contribuição de Felipe Pimentel Lopes de Melo ao trabalho e a contribuição de Rubens Queiroz pela identificação das espécies. Ventura D. agradece ao CNPq pela concessão da bolsa de mestrado.

\section{REFERÊNCIAS}

Adler P, Raff D, Lauenroth W. 2001. The effect of grazing on the spatial heterogeneity of vegetation. Oecologia, 128:465 $-479$.

Al-Seekh SH, Mohammad AG, Amro YA. 2009. Effect of Grazing on Soil Properties at Southern Part of West Bank Rangeland. Hebron University DSpace, 1:35-53.

Arévalo JR, Chinea E, Barquín E. 2007. Pasture management under goat grazing on Canary Islands. Agriculture, Ecosystems and Environmental, 118: 291-296.

Arévalo JR, De Nascimento L, Fernández-Lugo S, Mata J, Bermejo L. 2011. Grazing effects on species composition in different vegetation types (La Palma, Canary Islands). Acta Oecologica, 37:230-238.

Augustine DJ, Mcnaughton SJ. 1998. Ungulate effects on the functional species composition of plant communities: herbivore selectivity and plant tolerance. Journal of Wildlilfe Management, 62:1165-1183.

Baraza E, Gómez JM, Hódar JA, Zamora R. 2004. Herbivory has a greater impact in shade than in sun: response of Quercus pyrenaica seedlings to multifactorial environmental variation. Canadian Journal of Botany, 82:357-364. 
Barnes, ME. 2001. Effects of large herbivores and fire on the regeneration of Acacia erioloba woodlands in Chobe National Park, Botswana. African Journal of Ecology, 39:340-350.

Borcard D, Gillet F, Legendre P. 2011. Numerical Ecology with R. New York (NY), Springer, 319 p.

Couto AFAd', 2003. Dimensionamento do Mercado de Carne Ovina e Caprina no Brasil. In: II Simpósio Internacional Sobre Caprinos e Ovinos de Corte. Anais. João Pessoa, PB. p.71-81.

Chynoweth MW, Litton CM, Lepczyk CA, Hess SC, Cordell S. 2013. Biology and Impacts of Pacific Island Invasive Species. 9. Capra hircus, the Feral Goat (Mammalia: Bovidae). Pacific Science, 67:141-156.

Crawley MJ. 1997. Plant Ecology. Blackwell, Oxford.

Crawley, MJ. 1985. Reduction of oak fecundity by low-density herbivore populations. Nature, 314:163-164.

Crawley MJ. 2002. Statistical Computing: An Introduction to Data Analysis using S-Plus, Disponível em: http://www. amazon.com/dp/0471560405.

Devendra C, Burns CM. 1970. Goat Production in the Tropics (Revised Edn.). Technical Communication Bureaux of Animal Breeding and Genetics. Commonwealth Agriculture Bureau, England, p. 183.

Dregne, HE. 1986. Desertification of Arid Lands. Physics of Desertification, p. 4-34. Disponível em: http://www.ciesin. org/docs/002-193/002-193.html.

FAO, 1993. El papel de los animales domésticos en el control de la desertificación. PNUD/FAO, Oficina Regional de la Fao para America Latina y El Caribe, Santiago.

Fernández-Lugo S, Arévalo JR, De Nascimento L, Mata J, Bermejo LA. 2013. Long-term vegetation responses to different goat grazing regimes in semi-natural ecosystems: A case study in Tenerife (Canary Islands). Applied Vegetation Science, 16:74-83.

Forsyth DM, Coomes DA, Nugent G, Hall GMJ. 2002. Diet and diet preferences of introduced ungulates (Order: Artiodactyla). New Zealand Journal Zoology, 29:323-343.

Friedel, MM. 1986. The interaction with climate, soil and land use of Central Australian tree and shrub population. In: P.J.Joss, P.W. Lynch, and O.B. Williams (eds), Rangelands: a resource under siege. Proc. 2nd Internat. Rangeland Congress. Cambridge Univ. Press, Cambridge, U.K. p. 45-46.

Gibson, N, Kirkpatrick JB. 1989. Effects ofthe cessation of grazing on the grasslands and grassy woodlands of the Central Plateau, Tasmania. Australian Journal of Botany, 37:55-63.

Hakamata T, Hirashima T. 1978. Studies on nutrient cycles and fertilization of pasture. I. Outline of the cycles of nitrogen, phosphorus and potassium and model of nitrogen cycle. Japanese Society Grassland Science, 24:48-56.

Haridasan M. 1987. Distribution and mineral nutrition of aluminium-accumulating species in different plant communities of the cerrado region of the central Brazil. In: J.J. San Jose \& R. Montes (eds.). La Capacidad Bioreprodutiva de Sabanas. IVIC, Caracas, Venezuela, p. 309-348. 
Haynes RJ, Williams PH. 1993. Nutrient cycling and soil fertility in the grazed pasture ecosystem. Advances in Agronomy, 49:119-199.

Herrero M, Thornton PK, Gerber P, Reid RS. 2009. Livestock, livelihoods and the environment: understanding the tradeoffs. Current Opinion Environmental Sustainability, 1:111-120.

IBGE, 2010. Censo demográfico. 2000. Instituto Brasileiro de Geografia e Estatística, Rio de Janeiro.

INSA. 2012. Convivência com o semiárido. Instituto Nacional do Semiárido. Campina Grande, PB.

Jacomine PKT, Almeida JC, Medeiros LAR. 1973. Levantamento exploratório -reconhecimento de solos do Estado do Ceará. Recife. (DPP, Boletim Técnico, 28. SUDENE, Série Pedologia, 16), 2:830.

Jamelli D. 2015. Área de vida de caprinos domésticos (Capra hircus, bovidae) em uma paisagem de caatinga antropizada. Dissertação. Universidade Federal de Pernamubuco, Recife, PE.

King WB. 1985. Island birds: will the future repeat the past? In P. J. Moors, editor. Conservation of island birds. International Council for Bird Preservation. Cambridge. p. 3-15.

Landsberg J, Stol J Muller W. 1994. Telling the sheep (dung) from the goats. The Rangeland Journal, 16:122-134. https:// doi.org/10.1071/RJ9940122.

Leal IR, Vicente A, Tabarelli M. 2003. Herbivoria por caprinos na caatinga da região de xingó: uma análise preliminar. Ecologia e Conservação da Caatinga, p.695-716.

Legendre P, Legendre L. 1998. Numerical ecology.

Mantel N. 1967. The detection of disease clustering and a generalized regression approach. Cancer Research, 27:209-220.

Melo FPL, Martínez-Salas E, Benítez-Malvido J, G. Ceballos. 2010. Forest fragmentation reduces recruitment of largeseeded tree species in a semi-deciduous tropical forest of southern Mexico. Journal Tropical Ecology, 26:35.

Melo FPL. 2017. The socio-ecology of the caatinga: understanding how natural resource use shapes an ecosystem. In: Silva JMCD, Leal IR, Tabarelli M. (Eds.), Caatinga: The Largest Tropical Dry Forest Region in South America. Springer, Cham, Switzerland, p. 369-382.

Menezes T, Carmo R, Wirth R, Leal IR, Tabarelli M, Laurêncio A, Melo FPL. 2020. Introduced goats reduce diversity and biomass of herbs in Caatinga dry forest. Land degradation \& development. https://doi.org/10.1002/ldr.3693.

Moolman HJ, Cowling RM. 1994. The impact of elephant and goat grazing on the endemic flora of south African succulent thicket. Biological Conservation, 68:53-61.

MMA, 2014. Ministério do Meio Ambiente. Disponível em: https://www.mma.gov.br/biomas/caatinga. Acesso em dezembro de 2017.

Prado Júnior C. 1981. História econômica do Brasil. São Paulo: Brasiliense.

Pereira Filho, JM, Silva AM, A Cézar MF. 2013. Manejo da Caatinga para produção de caprinos e ovinos. Revista Brasileira de Saúde e Produção Animal, 14:77-90. 
Pickett STA, White PS. 1985. The ecology of natural disturbance and patch dynamics.

Pokorná P, Hejcmanová P, Hejcman M, Pavlu V. 2013. Activity time budget patterns of sheep and goats co-grazing on semi-natural species-rich dry grassland. Czech Journal Animal Science, 58:208-216.

Riet-Correa F, Bezerra CWC, Medeiros RMT. 2011. Plantas Tóxicas do Nordeste. Santa Maria: Sociedade Vicente Pallotti, 79p.

Rodal, MJN, Sales MF, Mayo SJ. 1998. Florestas serranas de Pernambuco: localização e diversidade dos remanescentes dos Brejos de Altitude. Recife, Imprensa Universitária da Universidade Federal Rural de Pernambuco.

Rodal MJN. 1992. Fitossociologia da vegetação arbustivo-arbórea em quatro áreas de caatinga em Pernambuco. Tese de doutorado, Universidade Estadual de Campinas, Campinas.

Rosenthal G, Schrautzer J, Eichberg C. 2012. Low intensity grazing with domestic herbivores: A tool for maintaining and restoring plant diversity in temperate Europe. Tuexenia, 32:167-205.

SADMET/INMET. 2014. Seção de Armazenamento de Dados meteorológicos/Instituto de Meteorologia. Disponível em: http://www.inmet.gov.br/html/central_servicos/combo_produtos.html>. Acesso em dezembro de 2018.

Sanderson EW, Redford KH, Vedder A, Coppolillo PB, Ward SE. 2002. A conceptual model for conservation planning based on landscape species requirements. Landscape and Urban Planning, 58:41-56.

Santos FCB, Souza BB, Alfaro CEP, César MF, Pimenta Filho E.C, Acosta AAA, Santos JRS. 2005. Adaptabilidade de caprinos exóticos e naturalizados ao clima semi-arido do nordeste brasileiro. Ciência e Agrotecnologia, 29:142-149.

Severson KE, Debano LF.1991. Influence of Spanish goats on vegetation and soils in Arizona chaparral. J. Range Management, 44:111-117.

Shepherd GJ. 2010. FITOPAC 2.1. Departamento de Botânica/UNICAMP.

Shi F, WU HY, Chen H, WU N. 2010. Effects of livestock exclusion on vegetation and soil properties under two topographic habitats in an alpine meadow on the eastern Qinghat - Tibetan Plateau. Polish Journal Ecology, 58:125-133.

Singh SP. 1998. Chronic disturbance, a principal cause of environmental degradation in developing countries. Environmental Conservation, 25:1-2.

SOCIEDADE NORDESTINA DE ECOLOGIA. 2002. Projeto Técnico para a Criação do Parque Nacional do Catimbau/ PE. Secretaria de Ciência, Tecnologia e Meio Ambiente de Penambuco - SECTMA, Recife.

Tabarelli M, Cardoso Da Silva JM, Gascon C. 2004. Forest fragmentation, synergisms and the impoverishment of neotropical forests. Biodiversidad and Conservation, 13:1419-1425.

Tedeschi LO, Cannas A, Fox DG. 2008. A nutrition mathematical model to account for dietary supply and requirements of energy and nutrients for domesticated small ruminants: The development and evaluation of the Small Ruminant Nutrition System. Revista Brasileira de Zootecnia, 37:178-190.

Ter Braak, CJF, Prentice IC. 1988. A Theory of Gradient Analysis. Advances Ecological Research, 18:271-317. 
Tsuiki M, WANG YS, Yiruhan Tsutsumi M, Shiyomi M. 2005. Analysis of grassland vegetation of the Southwest Heilongjiang steppe (China) using the power law. Journal of Integrative Plant Biology, 47:917-926.

World Resouces Institute - WRI. 1992. Estratégia global da biodiversidade. Kathleen Courrier, Cuiabá. 